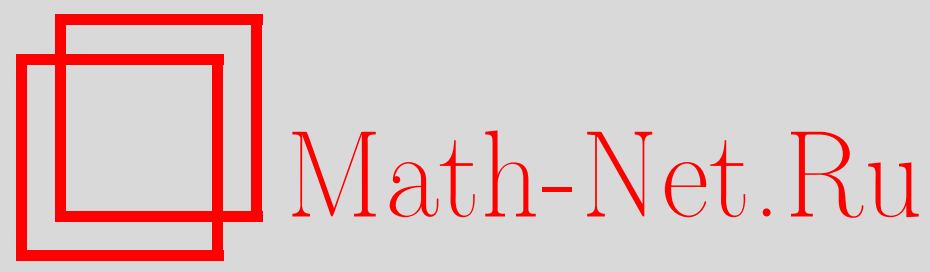

В. В. Подъяпольский, Полнота системы корневых функций одной нелокальной задачи в $L_{p}$, Матем. заметки, 2002, том 71, выпуск 6, 878-889

DOI: https://doi.org/10.4213/mzm392

Использование Общероссийского математического портала Math-Net.Ru подразумевает, что вы прочитали и согласны с пользовательским соглашением http://www.mathnet.ru/rus/agreement

Параметры загрузки:

IP: 44.207 .124 .84

26 апреля 2023 г., 18:02:09

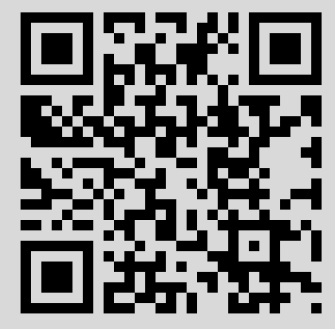




\section{ПОЛНОТА СИСТЕМЫ КОРНЕВЫХ ФУНКЦИЙ ОДНОЙ НЕЛОКАЛЬНОЙ ЗАДАЧИ В $L_{p}$}

\section{В.В. Подъяпольский}

Получены достаточные условия полноты системы корневых функций нелокальных эллиптических задач в пространствах Соболева $W_{p}^{l}(Q)$.

Библиографоия: 14 названий.

Введение. Спектральные свойства нелокальных задач для обыкновенных дифференциальных уравнений исследованы достаточно хорошо (см. [1]-[4] и др.). Например, в работе [3] для дифференциальных операторов второго порядка с нелокальными условиями установлены необходимые и достаточные условия базисности по Риссу системы корневых векторов.

Дискретность и угловая структура спектра нелокальных эллиптических задач, а также оценки резольвенты были установлены Скубачевским [5]. Однако асимптотика собственных значений, полнота системы корневых функций и суммируемость разложений по корневым функциям многомерных нелокальных задач остаются практически неисследованными. В связи с этим отметим работы Браудера [6], Моисеева [7], [8] и ряда других авторов. В [6] получен первьй член асимптотики спектральной функции и функции распределения собственньх значений самосопряженного в $L_{2}(Q)$ оператора, порожденного нелокальной эллиптической задачей. Однако условия существования такого оператора в работе [6] не были приведены, а нелокальные задачи, как правило, являются несамосопряженными. В работах [7] и [8] приведены примеры нелокальных задач для оператора Лапласа в двумерном круге, корневые функции которых полны и минимальны в $L_{2}(Q)$, но не образуют в нем базиса.

В предлагаемой статье рассматриваются свойства разложений по корневьм функциям нелокальных эллиптических задач. Вводятся операторы, действующие в пространствах Соболева $W_{q}^{s}(Q)$. При наложении некоторых условий все эти операторы имеют одну систему корневых функций, и таким образом устанавливается полнота этой системы функций в различных пространствах. Для доказательства используются методы статьи Лидского [9] и результаты об априорных оценках и разрешимости нелокальных эллиптических задач, полученные Ковалевой и Скубачевским в работах [5], [10].

Работа выполнена при финансовой поддержке Российского фонда фундаментальных исследований, грант № 01-01-01030, и Конкурсного центра по фундаментальным и естественным наукам при Санкт-Петербургском Государственном Университете, грант № 97-14.3-37. 
1. Рассмотрим уравнение

$$
A u(x)=f(x) \quad(x \in Q)
$$

с нелокальньми условиями

$$
B_{\mu} u=\left.\left(B_{\mu}^{0} u(x)+B_{\mu}^{1} u\right)\right|_{\partial Q}+B_{\mu}^{2} u=0 \quad(\mu=1, \ldots, m) .
$$

Здесь

$$
\begin{gathered}
A=A(x, \mathscr{D})=\sum_{|\alpha| \leqslant 2 m} a_{\alpha}(x) \mathscr{D}^{\alpha}, \\
B_{\mu}^{0}=B_{\mu}^{0}(x, \mathscr{D})=\sum_{|\alpha|=m_{\mu}} b_{\mu 0 \alpha}(x) \mathscr{D}^{\alpha}
\end{gathered}
$$

- дифференциальные операторы с комплексными коэффициентами $a_{\alpha}, b_{\mu 0 \alpha} \in C^{\infty}\left(\mathbb{R}^{n}\right)$, $n \geqslant 1 ; Q \subset \mathbb{R}^{n}$ - ограниченная область с границей $\partial Q \in C^{\infty}, \mathscr{D}^{\alpha}=\mathscr{D}_{1}^{\alpha_{1}} \ldots \mathscr{D}_{n}^{\alpha_{n}}$, $\mathscr{D}_{j}=-i \partial / \partial x_{j} ; f \in L_{p}(Q) ; m_{\mu} \leqslant 2 m-1, \mu=1, \ldots, m, p>1$. Условия на операторы $B_{\mu}^{1}, B_{\mu}^{2}$ будут приведены позже.

В дальнейшем также будут использоваться полиномы

$$
A^{0}(x, \xi)=\sum_{|\alpha|=2 m} a_{\alpha}(x) \xi^{\alpha}, \quad B_{\mu}^{0}(x, \xi)=\sum_{|\alpha|=m_{\mu}} b_{\mu 0 \alpha}(x) \xi^{\alpha}
$$

где $\xi=\left(\xi_{1}, \ldots, \xi_{n}\right), \xi^{\alpha}=\xi_{1}^{\alpha_{1}} \ldots \xi_{n}^{\alpha_{n}}$.

Введем локальную задачу, порожденную нелокальной задачей (1), (2),

$$
A u(x)=f(x) \quad(x \in Q)
$$

с краевыми условиями

$$
\left.B_{\mu}^{0}\right|_{\partial Q}=0 \quad(\mu=1, \ldots, m) .
$$

Если выполнены следующие условия, то будем говорить, что локальная задача (5), (6) эллиптична с параметром на луче $\arg \lambda=\theta$.

УСловиЕ I. Полином (по $\tau) A^{0}(x, \eta+\tau \nu)-\lambda$ имеет $m$ корней с положительными мнимыми частями при любых $x \in \bar{Q}, \nu \neq 0$ и $\eta \neq 0$, ортогональном к $\nu$, где $\arg \lambda=\theta$.

Из этого условия следует, что

$$
\arg A^{0}(x, \xi) \neq \theta
$$

для всех вещественных $\xi \neq 0$ и $x \in \bar{Q}$.

Пусть $x$-произвольная точка $\partial Q, \nu$ - единичньй вектор внутренней нормали и $\xi \neq 0$ произвольный касательньй вектор к $\partial Q$ в точке $x$. Обозначим через $\tau_{j}^{+}(x, \xi, \lambda)(j=1$, $\ldots, m)$ корни с положительньми мнимыми частями полинома по $\tau A^{0}(x, \xi+\tau \nu)-\lambda$, где $\lambda$ - произвольное число на луче $\arg \lambda=\theta$. 
УСЛОВИЕ II. Полиномы

$$
B_{\mu}^{0}(x, \xi+\tau \nu) \quad(\mu=1, \ldots, m)
$$

линейно независимы по модулю полинома

$$
\prod_{j=1}^{m}\left(\tau-\tau_{j}^{+}(x, \xi, \lambda)\right)
$$

для любых $x \in \partial Q$ и вектора $\xi$, касательного к $\partial Q$ в точке $x$, таких, что $|\xi|+|\lambda| \neq 0$.

Будем предполагать, что сушествуют $p_{1}, p_{2}\left(1<p_{1} \leqslant 2 \leqslant p_{2}\right)$ такие, что для всех $p \in\left[p_{1}, p_{2}\right]$ вьполнены следуюшие условия на нелокальные операторы.

УСловиЕ III. Линейные ограниченные операторы

$$
B_{\mu}^{1}: W_{p}^{m_{\mu}}(Q) \rightarrow L_{p}(Q), \quad B_{\mu}^{2}: W_{p}^{m_{\mu}+1 / p-r}(Q) \rightarrow L_{p}(\partial Q)
$$

таковы, что их сужения

$$
B_{\mu}^{1}: W_{p}^{4 m}(Q) \rightarrow W_{p}^{4 m-m_{\mu}}(Q), \quad B_{\mu}^{2}: W_{p}^{4 m-r}(Q) \rightarrow W_{p}^{4 m-m_{\mu}-1 / p}(\partial Q)
$$

- также ограниченные операторы и

$$
\begin{aligned}
\left\|B_{\mu}^{1} u\right\|_{0, p, Q} \leqslant c_{1}(p)\|u\|_{m_{\mu}, p, Q_{\delta}} & \left(u \in W_{p}^{m_{\mu}}(Q)\right), \\
\left\|B_{\mu}^{1} u\right\|_{4 m-m_{\mu}, p, Q} \leqslant c_{2}(p)\|u\|_{4 m, p, Q_{\delta}} & \left(u \in W_{p}^{4 m}(Q)\right),
\end{aligned}
$$

где $\|\cdot\|_{s, p, Q}$ - норма в пространстве Соболева $W_{p}^{s}(Q), Q_{\delta}=\{x \in Q: \rho(x, \partial Q)>\delta>0\}$, $0<r \leqslant m_{\mu}+1 / p(\mu=1, \ldots, m)$, а константы $c_{1}(p)$ и $c_{2}(p)$ не зависят от $u$.

Заметим, что из условий I и II следует выполнение условий 1)-3) в [10]. Из непрерывности полиномов следует, что выполнение условий I, II на луче $\arg \lambda=\theta$ означает выполнение тех же условий в некотором угле $|\arg \lambda-\theta|<\varepsilon$, где $\varepsilon$ достаточно мало.

ПримеР 1. Рассмотрим в ограниченной области $Q \subset \mathbb{R}^{n}$ с границей $\partial Q \in C^{\infty}$ уравнение

$$
A u(x)=-\Delta u(x)=f(x) \quad(x \in Q)
$$

с нелокальньп условием

$$
\left.B_{1} u\right|_{\partial Q}=\left.u\right|_{\partial Q}+\left.\sum_{j=1}^{l} a_{j}(x) u\left(\omega_{j}(x)\right)\right|_{\partial Q}=0
$$

где $a_{j} \in C^{\infty}(\partial Q), \omega_{j}$ - диффеоморфизмы класса $C^{\infty}$, отображающие окрестности гранищы $\partial Q$ в область $Q$. В данном случае $A^{0}(x, \xi)=\sum_{j=1}^{n} \xi_{j}^{2}, B_{1}^{0}(x, \xi)=1, B_{1}^{2}=0$. Нетрудно убедиться, что для локальной задачи, порожденной нелокальной задачей $(7)$, $(8)$, условия I, II выполнены на любом луче $\arg \lambda \neq 0$. Для нелокальной задачи $(7),(8)$ выполнено также условие III при любых $1<p_{1} \leqslant 2$. 
2. Обозначим через $W_{p}^{l}\left(Q,\left\{B_{\mu}\right\}_{1}^{m}\right)$ замыкание в $W_{p}^{l}(Q)$ множества функций в $C^{\infty}(\bar{Q})$, удовлетворяющих нелокальным условиям (2).

Введем неограниченньй оператор $\mathscr{A}_{s, p}$, действующий в $W_{p}^{s}(Q)\left(0 \leqslant s \leqslant 2 m, p_{1} \leqslant\right.$ $\left.p \leqslant p_{2}\right)$ следуюшим образом:

1) область определения оператора $\mathscr{D}\left(\mathscr{A}_{s, p}\right)=W_{p}^{2 m+s}\left(Q,\left\{B_{\mu}\right\}_{1}^{m}\right)$,

2) $\mathscr{A}_{s, p} u=A(x, \mathscr{D}) u\left(u \in \mathscr{D}\left(\mathscr{A}_{s, p}\right)\right)$.

Лемма 1. Пусть выполнено условие III при некоторых $p_{1}, p_{2}$ и локальная задача (5), (6) әллиптична с параметром на некотором луче $\arg \lambda=\theta$. Тогда $W_{p}^{2 m+s}\left(Q,\left\{B_{\mu}\right\}_{1}^{m}\right)$ плотно в $W_{p}^{s}(Q)$ при $0 \leqslant s<m_{\mu}+1 / p(\mu=1, \ldots, m), p_{1} \leqslant p \leqslant p_{2}$.

ДокАЗАтЕльство. Пусть $f$ - произвольная функция из $W_{p}^{s}(Q)$. Тогда для любого $p, p_{1} \leqslant p \leqslant p_{2}$, и $\varepsilon>0$ найдется такая $f_{p, \varepsilon} \in C^{\infty}(\bar{Q})$, что $\left\|f-f_{p, \varepsilon}\right\|_{s, p, Q}<\varepsilon$. В силу условия теоремы 1 из [10] (см. также [5]) найдется $q_{0}(p)>0$ такое, что отображение $\mathscr{L}: W_{p}^{2 m} \rightarrow W_{p}^{0}(Q, \partial Q)$, задаваемое по формуле

$$
\mathscr{L} u=\left(\left(A-q^{2 m} I\right) u, B_{1} u, \ldots, B_{m} u\right),
$$

обратимо при $\arg q=\theta /(2 m),|q|>q_{0}$, и выполняется оценка

$$
\|u\|_{2 m, p, Q} \leqslant c_{3}(p) \mid\|\mathscr{L} u\|_{0, p, Q, \partial Q} .
$$

Здесь $W_{p}^{0}(Q, \partial Q)=W_{p}^{0}(Q) \times \prod_{\mu=1}^{m} W_{p}^{2 m-m_{\mu}-1 / p}(\partial Q)$,

$$
\begin{gathered}
\|u\|_{l, p, \Omega}=\left\{\|u\|_{l, p, \Omega}^{2}+|q|^{2 l}\|u\|_{0, p, \Omega}^{2}\right\}^{1 / 2}, \\
\|f\|_{0, p, Q, \partial Q}=\left\{\left\|f_{0}\right\|_{0, p, Q}^{2}+\sum_{\mu=1}^{m}\left\|\mid f_{\mu}\right\|_{2 m-m_{\mu}-1 / p, p, \partial Q}^{2}\right\}^{1 / 2},
\end{gathered}
$$

$f=\left(f_{0}, f_{1}, \ldots, f_{m}\right), \lambda=q^{2 m}$.

Имеют место следующие интерполящионные неравенства (см. [11, предложение 2.2]):

$$
|q|^{2 m-s}\|u\|_{s, p, Q} \leqslant c_{s}\left(\|u\|_{2 m, p, Q}+|q|^{2 m}\|u\|_{0, p, Q}\right)
$$

когда $u \in W_{p}^{2 m}(Q), q \neq 0,0 \leqslant s<2 m$.

Положим $v_{p, \varepsilon}=\mathscr{L}^{-1}\left((A-\lambda I) f_{p, \varepsilon}, 0, \ldots, 0\right)$. Из теоремы 1 [10] следует, что $v_{p, \varepsilon} \in$ $W_{p}^{2 m+s}(Q)$. Для $f_{p, \varepsilon}-v_{p, \varepsilon}$ верна оценка (9). Следовательно,

$$
\begin{aligned}
|q|^{2 m-s}\left\|f_{p, \varepsilon}-v_{p, \varepsilon}\right\|_{s, p, Q} \leqslant & c_{4}\left(\sum_{\mu=1}^{m}\left\|B_{\mu} f_{p, \varepsilon}\right\| \|_{2 m-m_{\mu}-1 / p, p, \partial Q}^{2}\right)^{1 / 2} \\
= & c_{4}\left(\sum _ { \mu = 1 } ^ { m } \left(\left\|B_{\mu} f_{p, \varepsilon}\right\|_{2 m-m_{\mu}-1 / p, p, \partial Q}^{2}\right.\right. \\
& \left.\left.+|q|^{2\left(2 m-m_{\mu}-1 / p\right)}\left\|B_{\mu} f_{p, \varepsilon}\right\|_{0, p, \partial Q}^{2}\right)\right)^{1 / 2}, \\
\left\|f_{p, \varepsilon}-v_{p, \varepsilon}\right\|_{s, p, Q} \leqslant & c_{4}\left(\sum _ { \mu = 1 } ^ { m } \left(|q|^{2(s-2 m)}\left\|B_{\mu} f_{p, \varepsilon}\right\|_{2 m-m_{\mu}-1 / p, p, \partial Q}^{2}\right.\right. \\
& \left.\left.+|q|^{2\left(s-m_{\mu}-1 / p\right)}\left\|B_{\mu} f_{p, \varepsilon}\right\|_{0, p, \partial Q}^{2}\right)\right)^{1 / 2} .
\end{aligned}
$$

Тогда, выбирая $q$ достаточно большим, можно получить $\left\|f_{p, \varepsilon}-v_{p, \varepsilon}\right\|_{s, p, Q}<\varepsilon$. 
3. Подробнее рассмотрим случай $p=2$. Получим некоторые оценки резольвенты, необходимые нам в дальнейшем.

Предположим, что локальная задача (5), (6) эллиптична с параметром при некотором $0 \leqslant \theta<2 \pi$ и вьполнено условие III; тогда из теоремы 2.1 [5] следует, что оператор $\mathscr{A}_{s, 2}$ имеет дискретный спектр и резольвента оператора $\mathscr{A}_{s, 2}$

$$
R\left(\lambda, \mathscr{A}_{s, 2}\right)=\left(\lambda I-\mathscr{A}_{s, 2}\right)^{-1}
$$

может быть оценена сверху.

ЛЕмма 2. Пусть выполнено условие III и для некоторого $\theta$ выполнены условия I $u$ II. Тогда для достаточно больших $\lambda$, лежсащих на луче $\arg \lambda=\theta$, выполнена оиенка

$$
\left\|R\left(\lambda, \mathscr{A}_{s, 2}\right)\right\|_{s, 2}=O\left(|\lambda|^{s /(2 m)-1}\right),
$$

где под $\|\cdot\|_{s, 2}$ понимается норма оператора из $W_{2}^{s}(Q)$ в $W_{2}^{s}(Q)$.

ДоКАЗАТЕЛЬСТВо. Из условий леммы следует вьполнение условий 2.1-2.3 на луче $\arg q=\Theta /(2 m)$ в [5]. Тогда в силу теоремы 2.1 [5] для достаточно больших $q$ на этом луче выполнена оценка

$$
\|u\|_{2 m+s, Q} \leqslant c_{5}\|\mathscr{L} u\|_{s, Q, \partial Q} .
$$

Следовательно, если $u \in \mathscr{D}(\mathscr{A})$, то

$$
\begin{gathered}
\left|q^{2 m}\right| \cdot\|u\|_{s, 2, Q} \leqslant c_{6}\left(\left\|A u-q^{2 m} u\right\|_{s, 2, Q}+|q|^{s}\left\|A u-q^{2 m} u\right\|_{0,2, Q}\right) \\
\|u\|_{s, 2, Q} \leqslant c_{6} \frac{1+\|q\|^{s}}{|q|^{2 m}}\left\|A u-q^{2 m} u\right\|_{s, 2, Q}
\end{gathered}
$$

т.е.

$$
\|u\|_{s, 2, Q} \leqslant c_{7}|q|^{s-2 m}\left\|A u-q^{2 m} u\right\|_{s, 2, Q} .
$$

Отсюда и из теоремы 2.1 [5] следует утверждение леммы.

При доказательстве леммы 2 использовались разрешимость и априорная оценка при $s$, вообще говоря, нецельх, получаемые из результатов для целых $s$ путем интерполяции.

Пусть локальная задача (5), (6) эллиптична с параметром на некотором луче $\arg \lambda$ $=\theta$. Обозначим через $\lambda_{k}$ собственные значения оператора $\mathscr{A}_{s, 2}$. Пусть $z_{0}-$ некоторая фиксированная точка в резольвентном множестве оператора $\mathscr{A}_{s, 2}$. Тогда введем вполне непрерьвньй в $W_{2}^{s}(Q)$ оператор

$$
T_{s, 2}=\left(\mathscr{A}_{s, 2}-z_{0} I\right)^{-1}
$$

с собственными значениями $\mu_{k}=1 /\left(\lambda_{k}-z_{0}\right)$. Резольвенты операторов $\mathscr{A}_{s, 2}$ и $T_{s, 2}$ связаны следуюшим образом:

$$
R\left(\frac{1}{\lambda-z_{0}}, T_{s, 2}\right)=\left(\lambda-z_{0}\right) I-\left(\lambda-z_{0}\right)^{2} R\left(\lambda, \mathscr{A}_{s, 2}\right), \quad \lambda \neq \lambda_{k} .
$$

Очевидно, что операторы $T_{s, 2}$ и $\mathscr{A}_{s, 2}$ имеют одинаковые корневые функции. Поэтому в дальнейшем объектом изучения станет суммируемость разложений по корневьм функциям оператора $T_{s, 2}$.

Оператор $T_{s, 2}$ обладает следуюшим важным свойством. 
ЛЕмма 3. Пусть выполнено условие III и локальная задача (5), (6) әллиптична с параметром на некотором луче $\arg \lambda=\theta$. Тогда существует функиия $C(\lambda)$ такая, что для любого $\varepsilon>0$ выполнена оченка

$$
\left\|C(\lambda) R\left(\lambda, T_{s, 2}\right)\right\|_{s, 2} \leqslant \exp \left(|\lambda|^{-n /(2 m)-\varepsilon}\right) \quad\left(|\lambda| \leqslant \delta_{0}\right)
$$

где $C(\lambda)$ - иелая функиия по переменной $1 / \lambda$ порядка не выше $n /(2 m), \delta_{0}=\delta_{0}(\varepsilon)>0$.

ДокАЗАТЕЛЬСтво. В силу теоремы 2.1 [5] найдется такое $\lambda$, что $R\left(\lambda, \mathscr{A}_{s, 2}\right) f \in$ $W_{2}^{2 m+s}(Q)$ для любого $f \in W_{2}^{s}(Q)$. Тогда в силу тождества Гильберта

$$
T_{s, 2}=\left(\mathscr{A}_{s, 2}-z_{0} I\right)^{-1}=-R\left(\lambda, \mathscr{A}_{s, 2}\right)\left(I+\left(\lambda-z_{0}\right) R\left(z_{0}, \mathscr{A}_{s, 2}\right)\right)
$$

следовательно, $T_{s, 2} f \in W_{2}^{2 m+s}(Q)$ для любого $f \in W_{2}^{s}(Q)$. Применяя далее теорему А1.1 [12], получаем утверждение леммы.

Без ограничения общности можно предполагать, что $\lambda=0$ не является собственньм значением оператора $\mathscr{A}_{s, 2}$. Поэтому возьмем $z_{0}=0$.

Из (10) и (12) можно получить оценку нормы оператора $\left(I-\lambda T_{s, 2}\right)^{-1}$ на некоторых лучах.

ЛЕмма 4. Пусть выполнено условие III и для некоторого $\theta$ выполнены условия I $u$ II. Тогда для достаточно больших $\lambda$, лежсаших на луче $\arg \lambda=\theta$, выполнена оиенка

$$
\left\|\left(I-\lambda T_{s, 2}\right)^{-1}\right\|_{s, 2}=O\left(|\lambda|^{s /(2 m)}\right) .
$$

ДоказАтельство. Из (12) следует $\left(I-\lambda T_{s, 2}\right)^{-1}=I-\lambda R\left(\lambda, \mathscr{A}_{s, 2}\right)$. Тогда, применяя оценку $(10)$, получаем утверждение леммы.

4. Изучим полноту и базисность по Абелю системы корневых функций.

Пусть на лучах $\arg \lambda=\theta_{j}(j=1, \ldots, N)$ вьполнены условия I, II. Предположим также, что углы $L_{j}(j=1, \ldots, N)$, на которые разбивается комплексная плоскость этими лучами, имеют растворы меньше $2 m \pi / n$. В силу теоремы $1[10]$ в углах $\left|\arg \lambda-\theta_{j}\right|<\varepsilon$, где $\varepsilon$ достаточно мало, содержится конечное число собственных значений оператора $\mathscr{A}_{s, 2}$. Поэтому в них можно найти углы $\Lambda_{j}=\left\{\lambda: \theta_{j 1} \leqslant \arg \lambda \leqslant \theta_{j 2}\right\}$, состояшие из регулярньх точек оператора $\mathscr{A}_{s, 2}$. Очевидно, $\theta_{i j}$ можно выбрать так, что дополнение объединения $\Lambda_{j}$ состоит из углов, меньших $2 m \pi / n$. В силу того, что $\lambda=0$ не является собственньм значением $\mathscr{A}_{s, 2}$, найдется круг $B=\{\lambda:|\lambda| \leqslant r\}$ такой, что в нем нет ни одного собственного значения $\mathscr{A}_{s, 2}$. Обозначим через $G$ объединение $\Lambda_{j}$ и $B$ и через $\gamma$ - границу $G$. При интегрировании по $\gamma$ направление будет выбираться так, что $G$ остается слева. Без ограничения общности можно считать, что $\theta_{j}=\left(\theta_{j 1}+\theta_{j 2}\right) / 2$.

Пусть $\alpha=n /(2 m)+\varepsilon$, где $\varepsilon>0$ достаточно мало. Как хорошо известно, при $\alpha$ нецелых $\lambda=0$ - точка ветвления функции $\lambda^{\alpha}$ и две различные ветви этой аналитической функции отличаются на множитель $e^{2 k \pi i \alpha}$, где $k$ целое. Поэтому под $\lambda^{\alpha}$ будем понимать следующую функцию: в каждом из углов $L_{j}(j=1, \ldots, N)$ выберем такую ветвь функции $\lambda^{\alpha}$, что $\left|\arg \lambda^{\alpha}\right| \leqslant \pi / 2-\omega_{0}$, где $\omega_{0}>0$ для всех $\lambda \in L_{j}$. Это всегда можно сделать в силу малости $\varepsilon$. В дальнейшем будем считать, что $\varepsilon$ именно таково. 
Построим систему векторов $\left\{e_{q}\right\}(q=1,2, \ldots)$ из жордановых базисов в корневых подпространствах оператора $\mathscr{A}_{s, 2}$. В $[9$, гл. II, $\S 4]$ показано, что найдется система векторов $\left\{g_{j}\right\}(i=1,2, \ldots)$, биортогональная к системе $\left\{e_{q}\right\}$. Заметим, что из существования биортогональной системы следует минимальность системы $\left\{e_{q}\right\}$.

Приведем понятие суммирования рядов по методу Абеля, следуя работе [9]. Для произвольного вектора $f \in W_{2}^{s}(Q)$ рассмотрим формальньй ряд, строящийся следующим образом.

Задавшись некоторьп $\alpha>0$, введем в рассмотрение полиномы по переменной $t$

$$
P_{m}^{\alpha}\left(\xi^{-1}, t\right)=\frac{e^{t \xi^{-\alpha}}}{m !} \frac{d^{m}}{d \xi^{m}} e^{-t \xi^{-\alpha}} \quad(m=0,1, \ldots)
$$

Введем ряд

$$
\sum_{s=1}^{\infty} c_{s}(t) e_{s}
$$

коэффищиенты которого вычисляются следуюшим образом. Если $e_{q}-$ собственный вектор, не имеющий присоединенных, то

$$
c_{q}(t)=e^{-\lambda_{q}^{\alpha} t} c_{q}
$$

где $\lambda_{q}$ - собственное значение оператора $\mathscr{A}_{s, 2}$.

Если векторы $e_{q}, e_{q+1}, \ldots, e_{q+k}$ образуют жорданову цепочку, то

$$
c_{q+j}(t)=e^{-\lambda_{q}^{\alpha} t} \sum_{m=0}^{k-j} P_{m}^{\alpha}\left(\lambda_{q}, t\right) c_{q+j+m} \quad(0 \leqslant j \leqslant k)
$$

где

$$
c_{q}=\frac{\left(f, g_{q}\right)}{\left(e_{q}, g_{q}\right)}
$$

- коэффициенты разложения вектора $f$ по системе $e_{q}$.

Пусть ряд (16) обладает подпоследовательностью частичных сумм $S_{N_{\nu}}(t)$, которая сильно сходится в $W_{2}^{s}(Q)$ при всех $t>0$, и пусть $u(t)$ - соответствующая предельная функция:

$$
u(t)=\sum_{\nu=1}^{\infty}\left(\sum_{s=N_{\nu}+1}^{N_{\nu+1}} c_{s}(t) e_{s}\right)
$$

Пусть также

$$
\lim _{t \rightarrow+0} u(t)=f
$$

Будем говорить, что ряд (16), соответствующий $f$, суммируем $\kappa f$ nо метод $(A, \lambda, \alpha)$.

Будем также говорить, что система $e_{q}(q=1,2, \ldots)$ корневых функций оператора $\mathscr{A}_{s, 2}$ образует базис Абеля со скобками порядка $\alpha$ в $W_{2}^{s}(Q)$, если для любого $f \in$ $W_{2}^{s}(Q)$ соответствуюший ряд (16) суммируем к $f$ по методу $(A, \lambda, \alpha)$. 
Лемма 5. Ecлu $f=T_{s, 2} h, h \in W_{2}^{s}(Q) u 0 \leqslant s<2 m, m o$

$$
\lim _{t \rightarrow+0} u(t)=\lim _{t \rightarrow+0} \frac{1}{2 \pi i} \int_{\gamma} e^{-\lambda^{\alpha} t} T_{s, 2}\left(I-\lambda T_{s, 2}\right)^{-1} f d \lambda=f .
$$

ДокАЗАТЕЛЬСТво. Заметим, что

$$
\left(I-\lambda T_{s, 2}\right)^{-1} T_{s, 2}^{2}=\frac{1}{\lambda^{2}}\left[\left(I-\lambda T_{s, 2}\right)^{-1}-\left(I+\lambda T_{s, 2}\right)\right] .
$$

Поэтому, заменяя $f$ на $T_{s, 2} h$, получаем

$$
u(t)=\frac{1}{2 \pi i} \int_{\gamma} e^{-\lambda^{\alpha} t} \frac{\left(I-\lambda T_{s, 2}\right)^{-1} h}{\lambda^{2}} d \lambda-\frac{1}{2 \pi i} \int_{\gamma} e^{-\lambda^{\alpha} t} \frac{\left(I+\lambda T_{s, 2}\right) h}{\lambda^{2}} d \lambda .
$$

$\mathrm{B}$ силу выбора ветвей функции $\lambda^{\alpha}$ имеем $\left|\arg \lambda^{\alpha}\right| \leqslant \pi / 2-\omega_{0} ;$ кроме того, подынтегральная функция регулярна в пересечении $G$ с кругом $|\lambda|<R$ и достаточно быстро убывает в $\bar{G}$ при $R \rightarrow \infty$. Таким образом, второй интеграл в правой части равен нулю. Первый интеграл в силу оценки (14) сходится равномерно по $t$ при $t \geqslant 0$ и любом $s<2 m$. Поэтому

$$
\lim _{t \rightarrow+0} u(t)=\frac{1}{2 \pi i} \int_{\gamma} \frac{\left(I-\lambda T_{s, 2}\right)^{-1} h}{\lambda^{2}} d \lambda .
$$

Этот интеграл равен вычету подынтегральной функции при $\lambda=0$. Вычисляя его, находим

$$
\lim _{t \rightarrow+0} u(t)=T_{s, 2} h=f .
$$

Дословно повторяя доказательство леммы 6 из [9], можно получить следующее утверждение.

Лемма 6. Вычет функиии $e^{-\lambda^{\alpha} t} T_{s, 2}\left(I-\lambda T_{s, 2}\right)^{-1} f$ в полюсе $\lambda_{i}$ оператора $(I-$ $\left.\lambda T_{s, 2}\right)^{-1}$ равен

$$
-\sum_{q=q_{1}}^{q_{2}} c_{q}(t) e_{s}
$$

где $e_{q_{1}}, e_{q_{1}+1}, \ldots, e_{q_{2}}-$ жорданов базис оператора $T_{s, 2}$ в корневом подпространстве $\mathfrak{N}_{i}$, а $c_{q}(t)$ - коэффичиенты, вычисляемые по формулам (17), (18).

Используя приведенные результаты, получаем теорему о суммируемости по корневым функциям оператора $\mathscr{A}_{s, 2}$.

Теорема 1. Пусть $0 \leqslant s<2 m$, выполнено условие III для некоторых $p_{1}, p_{2} u$ существуют лучи $\arg \lambda=\theta_{j}, j=1, \ldots, N$, на комплексной плоскости такие, что

а) угль, на которые разбивается комплексная плоскость этими лучами, меньше, чем $2 m \pi / n$,

б) локальная задача (5), (6) әллиптична с параметром на лучах $\arg \lambda=\theta_{j}$, $j=1, \ldots, N$.

Тогда для любого $f \in W_{2}^{2 m+s}\left(Q,\left\{B_{\mu}\right\}_{1}^{m}\right)$ ряд по корневым функииям оператора $\mathscr{A}_{s, 2}$ суммируем $\kappa f$ в $W_{2}^{s}(Q)$ по методу $(A, \lambda, n /(2 m)+\varepsilon)$, где $\varepsilon>0$ достаточно мало. 
ДокАЗАТЕЛЬСтво. Для доказательства теоремы осталось показать, что для любого $t>0$ существует сходящаяся подпоследовательность частичных сумм. Согласно лемме 3 для резольвенты оператора $T_{s, 2}$ верна оценка (13). Пусть $R$ - некоторое положительное число и $1>\delta>0$. Используя теорему $11[13$, гл. I, $\S 8]$ при $\eta=\delta / 8$, получим, что в кольце

$$
R>|\lambda|>(1-\delta) R
$$

найдется окружность

$$
|\lambda|=\widetilde{R}
$$

на которой верна оценка

$$
|C(1 / \lambda)|>e^{-\ln \left\{\max \left|C\left(1 /\left(2 e \operatorname{Re}^{i \psi}\right)\right)\right|\right\}(2+\ln (12 e / \delta))} \quad(0 \leqslant \psi \leqslant 2 \pi) .
$$

Заменяя в оценке (13) $\varepsilon$ на $\varepsilon / 4$, имеем

$$
\left\|\left(I-\lambda T_{s, 2}\right)^{-1}\right\|_{s, 2} \leqslant e^{|\lambda|^{n /(2 m)+\varepsilon / 2}}
$$

на окружности (21).

Фиксируя $R$ и $\delta$, получим последовательность

$$
R_{\nu}=R(1-\delta)^{-\nu+1} \quad(\nu=0,1,2, \ldots) .
$$

Следовательно, в каждом кольце $R_{\nu}<|\lambda|<R_{\nu+1}$ найдется окружность $|\lambda|=\widetilde{R}_{\nu}$, на которой выполнена оценка (22). Обозначим через $\gamma_{\nu}$ гранищу пересечения кольца $\widetilde{R}_{\nu+1} \geqslant|\lambda| \geqslant \widetilde{R}_{\nu}$ с дополнением множества $G$, через $\gamma_{\nu, k}, k=1, \ldots, N,-$ связные компоненты пересечения окружности $|\lambda|=\widetilde{R}_{\nu}$ и дополнения $G$.

Пусть $N_{\nu}$ - число полюсов $\left(I-\lambda T_{s, 2}\right)^{-1}$ в круге $|\lambda| \leqslant \widetilde{R}_{\nu}$ с учетом их кратности. Тогда

$$
\frac{1}{2 \pi i} \int_{\gamma_{\nu}} e^{-\lambda^{\alpha} t} T_{s, 2}\left(I-\lambda T_{s, 2}\right)^{-1} f d \lambda=\sum_{s=N_{\nu}+1}^{N_{\nu+1}} c_{s}(t) e_{s} .
$$

На дугах $\gamma_{\nu k}$ интеграл оценивается следующим образом:

$$
\begin{aligned}
\left\|I_{\nu k}\right\|_{s, 2, Q} & =\left\|\frac{1}{2 \pi i} \int_{\gamma_{\nu k}} e^{-\lambda^{\alpha} t} T_{s, 2}\left(I-\lambda T_{s, 2}\right)^{-1} f d \lambda\right\|_{s, 2, Q} \\
& \leqslant\left\|T_{s, 2}\right\|_{s, 2}|\lambda| e^{-t \operatorname{Re} \lambda^{\alpha}} e^{|\lambda|^{n /(2 m)+\varepsilon / 2}}\|f\|_{s, 2, Q} \quad(k=1, \ldots, N) .
\end{aligned}
$$

По построению функции $\lambda^{\alpha}$ на каждой дуге $\left|\arg \lambda^{\alpha}\right|<\pi / 2-\omega_{0}$, поэтому

$$
\operatorname{Re} \lambda^{\alpha} \geqslant|\lambda|^{\alpha} \sin \omega_{0} .
$$

Учитьвая, что

$$
R_{\nu}<\widetilde{R}_{\nu}<R_{\nu+1}=R(1-\delta)^{-\nu}
$$

получим

$$
\left\|I_{\nu k}\right\|_{s, 2, Q} \leqslant\left\|T_{s, 2}\right\|_{s, 2} R_{\nu} e^{-t\left|R_{\nu}\right|^{n /(2 m)+\varepsilon} \sin \omega_{0}+\left|R_{\nu}\right|^{n /(2 m)+\varepsilon / 2}}\|f\|_{s, 2, Q} \quad(k=1, \ldots, N) .
$$


Аналогично оцениваются интегралы на прямолинейных участках контура $\gamma_{\nu}$ :

$$
\left\|J_{\nu k}\right\|_{s, 2, Q} \leqslant\left\|T_{s, 2}\right\|_{s, 2} R_{\nu}^{1+s /(2 m)} e^{-t R_{\nu}^{\alpha} \sin \omega_{0}}\left((1-\delta)^{-2}-1\right)\|f\|_{s, 2, Q} \quad(k=1, \ldots, 2 N) .
$$

Из оценок (23) и (24) видно, что ряд

$$
\sum_{\nu=1}^{\infty}\left(\sum_{k=1}^{N}\left(\left\|I_{\nu k}\right\|_{s, 2, Q}+\left\|I_{\nu+1, k}\right\|_{s, 2, Q}\right)+\sum_{k=1}^{2 N}\left\|J_{\nu k}\right\|_{s, 2, Q}\right)
$$

сходится при любом $t>0$.

СЛЕДСТВИЕ 1. Пусть выполнены условия теоремы $1 u 0 \leqslant s<m_{\mu}+1 / 2$, $\mu=1, \ldots, m$. Тогда система корневых функиий оператора $\mathscr{A}_{s, 2}$ образует в $W_{2}^{s}(Q)$ базис Абеля со скобками порядка $n /(2 m)+\varepsilon$, әде $\varepsilon>0$ - достаточно мало.

ДокАЗАтЕльство. В силу леммы $1 W_{2}^{2 m+s}\left(Q,\left\{B_{\mu}\right\}_{1}^{m}\right)$ плотно в $W_{2}^{s}(Q)$. Тогда из оценок $(23),(24)$ следует, что $u(t)$ существует для любого $f \in W_{2}^{s}(Q)$. Введем семейство операторов $U(t) f(t>0)$, действующих в $W_{2}^{s}(Q)$ по формуле $U(t) f=u(t)$. В силу теоремы Банаха-Штейнгауза и леммы 1 получаем, что семейство операторов $U(t)$ $(0<t \leqslant \delta)$ для некоторого $\delta>0$ равномерно ограничено в $W_{2}^{s}(Q)$. Тогда, выбирая $f_{\varepsilon} \in W_{2}^{2 m+s}\left(Q,\left\{B_{\mu}\right\}_{1}^{m}\right)$ такой, что $\left\|f_{\varepsilon}-f\right\|_{s, 2, Q}<\varepsilon$, имеем

$$
\begin{aligned}
\|u(t)-f\|_{s, 2, Q} & =\left\|U(t) f-U(t) f_{\varepsilon}+U(t) f_{\varepsilon}-f_{\varepsilon}+f_{\varepsilon}-f\right\|_{s, 2, Q} \\
& \leqslant\|U(t)\|_{s, 2}\left\|f-f_{\varepsilon}\right\|_{s, 2, Q}+\left\|U(t) f_{\varepsilon}-f_{\varepsilon}\right\|_{s, 2, Q}+\left\|f-f_{\varepsilon}\right\|_{s, 2, Q}
\end{aligned}
$$

где $0<t \leqslant \delta$. В силу того, что $\lim _{t \rightarrow 0} U(t) f_{\varepsilon}=f_{\varepsilon}$, получаем при достаточно мальх $t>0$, что

$$
\|u(t)-f\|_{s, 2, Q}<\varepsilon
$$

СлЕДСТВИЕ 2. Пусть выполнены условия теоремы 1. Тогда система корневых функиий оператора $\mathscr{A}_{s, 2}$ полна в $W_{2}^{2 m+s}\left(Q,\left\{B_{\mu}\right\}_{1}^{m}\right)$ в топологии $W_{2}^{2 m+s}(Q)$.

ДокАЗАТЕЛЬСТво. В силу теоремы 1 система корневых функций оператора $\mathscr{A}_{s, 2}$ плотна в $W_{2}^{2 m+s}(Q)$ в топологии $W_{2}^{s}(Q)$. Без ограничения общности можно считать, что $\mathscr{A}_{s, 2}^{-1}$ существует и отображает ограниченным образом $W_{2}^{s}(Q)$ в $W_{2}^{2 m+s}\left(Q,\left\{B_{\mu}\right\}_{1}^{m}\right)$. Тогда для произвольного $f \in W_{2}^{2 m+s}\left(Q,\left\{b_{\mu}\right\}_{1}^{m}\right)$ вектор $g=\mathscr{A}_{s, 2} f \in W_{2}^{s}(Q)$. В силу полноты системы корневых функций оператора $\mathscr{A}_{s, 2}$ найдется последовательность $\left\{\psi_{k}\right\}$ конечных сумм корневых функций такая, что $\psi_{k} \rightarrow g$. Тогда

$$
\mathscr{A}_{s, 2}^{-1} \psi_{k} \rightarrow \mathscr{A}_{s, 2}^{-1} g=f \quad(k \rightarrow \infty)
$$

в топологии $W_{2}^{2 m+s}(Q)$. Остается только заметить, что корневые функции инвариантны относительно $\mathscr{A}_{s, 2}^{-1}$.

ЗАмЕчАнИЕ 1. Предположим, что локальная задача (5), (6) эллиптична с параметром на каком-нибудь луче и вьполнено условие III при некоторьх $p_{1}$ и $p_{2}$. Тогда, рассуждая аналогично работе [12, с. 131-132], нетрудно получить, что операторы $\mathscr{A}_{s, p}$ при $p_{1} \leqslant p \leqslant p_{2}, 0 \leqslant s \leqslant 2 m$ имеют одинаковые корневые функции. Таким образом, рассматривая системы корневых функций операторов $\mathscr{A}_{s, p}$, мы рассматриваем одну и ту же систему функций. Обозначим ее как $\mathscr{H}_{p_{1} p_{2}}$. Очевидно также, что операторы $\mathscr{A}_{s, p}$ имеют одинаковые собственные значения. 
Теорема 2. Пусть выполнены условия теоремы 1. Тогда система функиий $\mathscr{H}_{p_{1} p_{2}}$ полна в $W_{p}^{s}(Q)$ и в $W_{p}^{2 m+s}\left(Q,\left\{B_{\mu}\right\}_{1}^{m}\right)$ в топологии $W_{p}^{2 m+s}(Q)$ при $p_{1} \leqslant$ $p \leqslant p_{2}, 0 \leqslant s<m_{\mu}+1 / p, \mu=1, \ldots, m$.

ЗАмЕчаниЕ 2. В случае, когда локальная задача (5), (6), порожденная нелокальной задачей $(1),(2)$, эллиптична с параметром на любом луче $\arg \lambda=\theta \neq 0$, утверждения о суммируемости и базисности по Абелю могут быть усилены следующим образом: вместо порядка $\alpha=\beta_{0}+\varepsilon$, где $\varepsilon>0$ достаточно мало, можно взять любое $\alpha>\beta_{0}$. Кроме этого, для таких задач можно получить первый член асимптотики собственных значений. В силу замечания 1 полученная асимптотика собственных значений будет выполняться для всех операторов $\mathscr{A}_{s, p}$, где $p_{1} \leqslant p \leqslant p_{2}, 0 \leqslant s \leqslant 2 m$.

Имеет место следующая

ТЕОремА 3. Пусть выполнено условие III при некоторых $p_{1}, p_{2}$ и на любом луче $\arg \lambda=\theta \neq 0$ локальная задача (5), (6) әллиптична с параметром. Тогда для функиии распределения модулей собственных значений операторов $\mathscr{A}_{s, p}-N(|\lambda|)-$ справедлива следующая асимптотическая формула:

$$
N(|\lambda|) \sim \omega(\mathscr{A}, Q)|\lambda|^{n /(2 m)} \quad(\lambda \rightarrow+\infty),
$$

əде

$$
\omega(\mathscr{A}, Q)=\frac{1}{(2 \pi)^{n}} \int_{Q} \int_{\left\{\xi \in \mathbb{R}^{n}: A^{0}(x, \xi) \leqslant 1\right\}} d \xi d x,
$$

$p_{1} \leqslant p \leqslant p_{2}, 0 \leqslant s \leqslant 2 m$.

ДокАЗАТЕЛЬСТво. В силу условия теоремы и леммы 2 при $s=0$ на любом луче $\arg \lambda=\theta \neq 0$ выполнена оценка

$$
\left\|R\left(\lambda, \mathscr{A}_{0,2}\right)\right\|_{0,2}=O\left(|\lambda|^{-1}\right)
$$

Применяя теорему 1 [14], получаем доказьваемое утверждение для оператора $\mathscr{A}_{0,2} . \mathrm{B}$ силу замечания 1 результат остается верным и для всех операторов $\mathscr{A}_{s, p}$.

ПРИмеР 2. Рассмотрим следующую нелокальную краевую задачу для оператора Лапласа в двумерном единичном круге $K$

$$
\begin{gathered}
A v(x)=-\Delta v(x)=f(x) \quad(x \in K), \\
B v=v\left(\frac{1}{2}, \varphi\right)-v(1, \varphi)=0, \quad \varphi \in[0,2 \pi] .
\end{gathered}
$$

Вводя операторы $\mathscr{A}_{s, p}$ и применяя теорему 2 , получаем, что система корневых функций $\mathscr{A}_{s, p}$ полна в $W_{p}^{s}(Q)$ и в $W_{p}^{2+s}(Q, B)$ в топологии $W_{p}^{2+s}(Q)$ при $p>1,0 \leqslant s<1 / p$. В силу теоремы 3 для собственных значений оператора $\mathscr{A}_{s, p}$ верна асимптотическая формула

$$
N(r) \sim \frac{1}{4} r \quad(r \rightarrow+\infty)
$$

Автор выражает благодарность А. Л. Скубачевскому за постоянное внимание к работе. 


\section{СПИСОК ЦИТИРОВАННОЙ ЛИТЕРАТУРЫ}

[1] Тамаркин Я. Д. О некоторых общих задачах теории обыкновенных линейных дифференциалных уравнений и о разложении произволных функций в ряды. Петроград: Типография М. П. Фроловой, 1917.

[2] Krall A. M. The development of general differential and general boundary systems // Rocky Mountain J. Math. 1972. V. 5. P. 493-542.

[3] Ильин В.А. Необходимые и достаточные условия базисности Рисса корневых векторов разрывных операторов второго порядка // Дифференц. уравнения. 1986. Т. 22. № 12 . C. $2059-2071$.

[4] Шкаликов А. А. О базисности собственных функций обыкновенных дифференциальных операторов с интегральными краевыми условиями // Вестн. МГУ. Сер. 1. Матем., мех. 1982. № 6. C. $12-21$.

[5] Скубачевский А. Л. Нелокальные эллиптические задачи с параметром // Матем. сб. 1983. T. 121. №6. C. 201-210.

[6] Browder F. Asymptotic distribution of eigenvalues and eigenfunctions for nonlocal elliptic boundary value problems // Amer. J. Math. 1965. V. 87. № 1. P. 175-195.

[7] Моисеев Е.И. О спектральных характеристиках одной нелокальной краевой задачи // Дифференц. уравнения. 1994. Т. 30. № 5. С. 864-872.

[8] Моисеев Е.И. Об отсутствии свойства базисности у системы корневых функций одной нелокальной краевой задачи // Дифференц. уравнения. 1994. Т. 30. № 12. С. 2082-2093.

[9] Лидский В.Б. О суммируемости рядов по главным векторам несамосопряженных операторов // Тр. ММО. 1962. Т. 11. С. 3-35.

[10] Ковалева О. А., Скубачевский А. Л.Нелокальные эллиптические задачи в пространствах Соболева $W_{p}^{l}(Q) / /$ Докл. РАН. 1999. Т. 366. № 4. С. 449-451.

[11] Agranovich M., Denk R., Faierman M. Weakly Smooth Nonselfadjoint Spectral Elliptic Boundary Problems. Advances in Partial Differential Equations (Math. Topics, 14). Berlin: Akademie Verlag, 1997.

[12] Agmon S. On the eigenfunctions and on the eigenvalues of general elliptic boundary value problems // Comm. Pure Appl. Math. 1962. V. 15. P. 119-147.

[13] Левин Б.Я. Распределение корней целых функций. М.: ГИТТЛ, 1956.

[14] Бойматов К.Х., Костюченко А. Г. Спектральная асимптотика несамосопряженных эллиптических систем дифференциальных операторов в ограниченных областях // Матем. сб. 1990. Т. 181. № 12. С. $1678-1693$. 